In the history of physics, numerous attempts have been made to explain the origin of the solar system and the universe. Many earlier theories on these problems were rejected after repeated analysis or comparison with later astronomical data. Alfvén's theory on the origin of the solar system is one of the attempts that are still considered possible solutions of these problems. It is based on the ionization processes and plasma physical phenomena occurring when a cosmic cloud of neutral gas falls through a plasma cloud towards a magnetized central body which represents the sun. As an important feature of the theory a violent ionization process is assumed to take place when the motion of the two clouds through each other approaches the velocity corresponding to the ionization energy. This 'critical velocity' has different values for different elements. A separation of matter is therefore expected to take place and should be evident in the chemical composition of the planets. Several laboratory experiments have confirmed the existence of the critical velocity. Its significance extends far beyond the problem of the origin of the solar system.

An early cosmological theory by Klein has been further developed since 1962 by Alfvén and Klein. It is characterized by the hypothesis that the universe as a whole consists of equal amounts of matter and antimatter. The main contribution by Alfvén is the introduction of the concept 'ambiplasma' which comprises matter and antimatter.

Since 1940 the development in plasma physics has resembled an explosion and has strongly modified important parts of cosmical physics and opened promising fields of application, such as thermonuclear fusion. To a large extent this progress was initiated by Hannes Alfvén and, in any case, no other research worker in this field has had a comparable influence on its development. Basic concepts and results due to Alfvén, already now to be regarded as classical, are the equivalent magnetic moment, the 'frozen' magnetic field lines, the Alfvén velocity of magnetohydromagnetic waves and the critical velocity for the interaction between a plasma and a neutral gas. These achievements are a monument to Alfvén's brilliance and working methods, so manysided and rich in ideas, and characterized by the ability of co-ordinating complicated systems and problems to form a logical and aesthetic entity.

B. Lehnert

\title{
EPS Individual Ordinary Member No. 1000
}

In the autumn of last year, just when the EPS was two years old, the 1000th Individual Ordinary Member joined the Society. He is Dr. Ing. Dipl. Phys. Hubert Patalong of Munich, a longstanding member of the German Physical Society. During a recent interview Mr. Patalong kindly told us about his background and outlined what prompted him to join and what he hopes the EPS might help to achieve.

Since leaving the University of Frankfurt in 1952 he has been working at research laboratories of Siemens A.G., engaged on what he terms 'development' rather than 'pure research', and, while there, he obtained his doctorate under M. Kersten at Aachen. He manages to keep in touch with the academic world by lecturing at Erlangen University.

When he received the circular in which the President K. Ganzhorn appealed to all members of the German Physical Society to support the EPS by joining as Individual Ordinary Members (see Europhysics News No. 11) his response was immediate. What impressed him in this appeal was the idea that physicists are particularly qualified to contribute to a better understanding between peoples because their scientific interests transcend political and national boundaries. The agreement on a common language (English) as a working medium for the Society, he feels, is a remarkable and gratifying achievement. In helping to bring together physicists of different nationality but common interests, the EPS can greatly promote the spiritual unification of Europe. Having lived through a period of violence and destruction engendered by strife between nations and witnessed the splitting of his country, Mr. Patalong welcomes particularly the increasing contacts in science between East and West encouraged by the EPS. He strongly supports the Society's endeavours to improve the coordination of research, which incidentally could bring about greater freedom of movement for physicists between their centres of activity. Working in industry

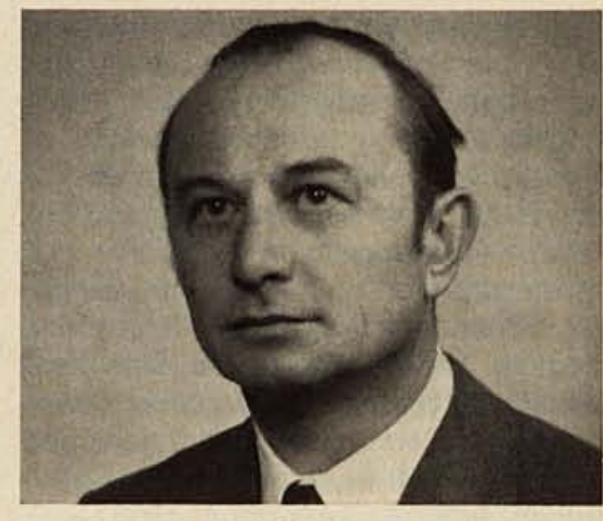

the scientist often lacks the opportunities of his academic colleagues. Attendance at scientific conferences to make vital contacts with co-workers in the field is difficult, and time is always too short to read even essential journals. The EPS should help to restore to the industrial physicist. who often feels forgotten, the sense of belonging to the great community of physicists.

We hope that the EPS will be able to meet these expectations fully.

\section{Classified Advertisement}

\section{Proceedings of the International Conference on Magnetism Grenoble, 14-19 September 1970}

These Proceedings are published in the 'Journal de Physique' and comprise 48 invited papers by leading experts on the latest results concerning fundamental problems in magnetism, as well as 475 contributed papers with recent original results regarding experiment and theory. The papers cover classical subjects, such as anisotropy and the mechanism of magnetization, and also new subjects such as Kondo effect, metal-insulator transition, etc.

About 1200 pages, $21 \times 29.7 \mathrm{~cm}$ Publication date: February-March 1971 Price: US \$ 30

Journal de Physique

33, rue Croulebarbe, $F$ - 75 PARIS $13 e$ 